\title{
NOTIZEN
}

\section{Der Informationsverlust in der Theorie der Kleinwinkelvielfachstreuung}

\section{VALENTIN ERNST*}

Institut für Theoretische Physik der Universität München

(Z. Naturforschg. 21 a, 359-360 [1966] ; eingegangen am 27. August 1965)

Die Theorie der elastischen Kleinwinkelvielfachstreuung ${ }^{1}$ stellt sich die Aufgabe, aus dem als bekannt vorausgesetzten elastischen differentiellen Wirkungsquerschnitt $\sigma(\cos \Theta)$ (kugelsymmetrisches Potential), der Dicke $d$ und Dichte $N$ (= Anzahl der statistisch isotrop verteilten Streuzentren in der Raumeinheit) der Streuschicht und ggf. aus der Richtungsverteilung $I^{\text {in }}(\boldsymbol{n})$ ( $\boldsymbol{n}$ bezeichnet den Richtungseinheitsvektor) der auf die Streufolie auftreffenden Teilchen die Verteilung $I^{\text {out }}(\boldsymbol{n})$ der austretenden Intensität zu berechnen. Es wurde auch die Frage aufgeworfen, ob man nicht aus den unmittelbar meßbaren Größen $I^{\text {in }}(\boldsymbol{n}), I^{\text {out }}(\boldsymbol{n})$, $N, d$, den Wirkungsquerschnitt $\sigma(\cos \Theta)$ berechnen könne; Cormack ${ }^{2}$ gab Formeln an, die eine solche „Umkehrung“ der Theorie zu ermöglichen scheinen. Gegen eine solche Möglichkeit spricht aber der mit dem statistischen Prozeß der Vielfachstreuung notwendig verknüpfte Informationsverlust, der eine vollständige Bestimmung von $\sigma(\cos \Theta)$ außer im Falle sehr dünner Streufolien, wo die Einfachstreuung dominiert, sicher unmöglich macht. Die „Umkehrung“ der Theorie muß deshalb aus der Antwort auf die Frage bestehen, welche Teilinformation man über den differentiellen Wirkungsquerschnitt aus den angeführten, direkt meßbaren Größen bei Vorliegen von Mehr- oder Vielfachstreuung gewinnen könne. Wir wollen hier eine Antwort auf diese Frage an Hand einer Verallgemeinerung der Goudsmit-Saundersonschen Form ${ }^{3}$ der Theorie der Kleinwinkelvielfachstreuung skizzieren.

Bezeichnet $\sigma_{0}$ im Einklang mit der späteren Definition (5) den totalen Wirkungsquerschnitt und stellt

$$
\begin{aligned}
F_{0}(\boldsymbol{n}): & =I^{\text {in }}(\boldsymbol{n}), \\
F_{n}(\boldsymbol{n}): & =\frac{1}{\sigma_{0}} \int_{\Omega^{\prime}} \mathrm{d} \boldsymbol{n}^{\prime} F_{n-1}\left(\boldsymbol{n}^{\prime}\right) \sigma\left(\boldsymbol{n} \boldsymbol{n}^{\prime}\right), \\
n &
\end{aligned}
$$

wobei die Integration über die ganze Oberfläche der Einheitskugel $\Omega^{\prime}$ der Richtungen $n^{\prime}$ zu erstrecken ist, die rekursive Definition der $n$-fachen Faltung von $I^{\text {in }}(\boldsymbol{n})$ mit dem normierten Wirkungsquerschnitt

$$
\sigma(\cos \Theta) / \sigma_{0}=\sigma\left(\boldsymbol{n} \boldsymbol{n}^{\prime}\right) / \sigma_{0}
$$

* Jetzt: Department of Physics, University of Pittsburgh, Pittsburgh, $\mathrm{Pa}$.

1 Eine Übersicht und weitere Literatur: W. T. Scotr, Rev. Mod. Phys. 35, 231 [1963]. dar, so ist $I^{\text {out }}(\boldsymbol{n})$ unter Voraussetzung ungestörter, rein elastischer Potentialeinzelstreuakte offensichtlich durch die Formel

$$
I^{\text {out }}(\boldsymbol{n})=\sum_{n=0}^{\infty} w_{n} F_{n}(n), \quad \sum_{n=0}^{\infty} w_{n}=1
$$

gegeben, wobei $w_{n} \geqq 0$ die Wahrscheinlichkeit dafür ist, daß ein Teilchen des Strahles im Streugebiet genau $n$-mal gestreut wird. Zur Theorie der Kleinwinkelvielfachstreuung kommen wir über die einzige weitere Annahme, daß $w_{n}$ durch die Porsson-Verteilung

$$
w_{n}=\frac{M^{n}}{n !} e^{-M}, \quad M:=N d \sigma_{0}
$$

approximiert werden darf. Entwickelt man $I^{\text {in }}(\boldsymbol{n})$, $I^{\text {out }}(\boldsymbol{n})$ und $\sigma(\cos \Theta)$ nach normierten Kugelflächenfunktionen $Y_{l}^{m}(\boldsymbol{n})$ und nach Legendreschen Polynomen $P_{l}(\cos \Theta)$ gemäß

$$
\begin{array}{r}
I^{\text {in, out }}(\boldsymbol{n})=\sum_{l=0}^{\infty} \sum_{m=-l}^{+l} I_{l m}^{\text {in, out }} Y_{l}^{m}(\boldsymbol{n}), \\
\sigma(\cos \Theta)=\sigma\left(\boldsymbol{n} \boldsymbol{n}^{\prime}\right)=\sum_{l=0}^{\infty} \frac{2 l+1}{4 \pi} \sigma_{l} P_{l}\left(\boldsymbol{n} \boldsymbol{n}^{\prime}\right) \\
=\sum_{l=0}^{\infty} \sum_{m=-l}^{+l} \sigma_{l} Y_{l}^{m}\left(\boldsymbol{n}^{\prime}\right) \overline{Y_{l}^{m}}(\boldsymbol{n}),
\end{array}
$$

wobei der Querstrich den Ubergang zum KonjugiertKomplexen anzeigt, und trägt man (3), (4) und (5) unter Berücksichtigung von (1) in (2) ein, so findet man

$$
I_{l m}^{\text {out }}=I_{l m}^{\text {in }} \exp \left\{-N d\left(\sigma_{0}-\sigma_{l}\right)\right\} .
$$

Diese Beziehung liefert für $\delta$-förmige, normal auf die Streufolie auftreffende Intensitätsverteilung die Formeln von Goudsmit und Saunderson ${ }^{3}$ und kann auch durch Verallgemeinerung der Herleitung von LEwIS ${ }^{4}$ gewonnen werden.

Da $I^{\text {in }}(\boldsymbol{n})$ und $I^{\text {out }}(\boldsymbol{n})$ direkt meßbar sind, können die $l_{m}^{\text {n }}$ und $I_{l m}^{\text {out }}$ durch Umkehrung von (4) und aus ihnen nach (6) die Differenzen $\sigma_{0}-\sigma_{l}$ bestimmt werden. Da wir als Regelfall $\sigma_{l} \rightarrow 0$ für $l \rightarrow \infty$ voraussetzen dürfen, erhalten wir für $\sigma_{0}$ die Formel

$$
\sigma_{0}=\lim _{l \rightarrow \infty} \frac{1}{N d} \ln \left(I_{l m}^{\text {in }} / I_{l m}^{\text {out }}\right),
$$

so daß wir scheinbar die $\sigma_{l}$ und mit ihnen nach (5) den Wirkungsquerschnitt $\sigma(\cos \Theta)$ bestimmen können.

2 A. M. Cormack, Nucl. Phys. 52, 286 [1964].

3 S. Goudsmit u. J. L. Saunderson, Phys. Rev. 57, 24 [1940] ; 58, 36 [1940].

4 H. W. Lewis, Phys. Rev. 78, 526 [1950]. 
Falls für jedes $l$ für mindestens einen Wert von $m$ die Beziehung $I l_{m}^{\text {in }} \neq 0$ gilt, z. B. wenn $I^{\text {in }}(\boldsymbol{n})$ die Form einer $\delta$-Funktion für die Richtung normal zur Streufolie hat, so scheint eine solche Umkehrung im rein mathematischen Sinne tatsächlich möglich zu sein. Man kann aber vom physikalischen Standpunkt aus jene $I^{\text {in }}(\boldsymbol{n})$ nicht von vornherein diskriminieren, bei denen die $l_{l m}^{\text {in }}$ für einzelne $l$ oder für alle $l$ ab einem $l_{0}$ exakt verschwinden. Dann können über die entsprechenden $\sigma_{0}-\sigma_{l}$ aus (6) keine Aussagen gewonnen werden und der Grenzwert (7) bleibt unter Umständen unbestimmbar. In diesen Fällen läßt sich die Reihe (5) nicht aufsum. mieren, da endliche Summanden unbestimmt bleiben.

In der physikalischen Praxis muß eine solche Nichtumkehrbarkeit als Regelfall angesehen werden, weil man zu berücksichtigen hat, daß $I^{\text {in }}(\boldsymbol{n})$ und $I^{\text {out }}(\boldsymbol{n})$ mit einem nicht beliebig kleinen Meßfehler $\varepsilon$ versehen sind, so daß die Reihen (4) abgebrochen werden müssen, wenn der mathematische Approximationsfehler den Meßfehler $\varepsilon$ unterschreitet. Brechen so die Reihen (4) bei $l=l_{\varepsilon}$ in, out ab und ist $l_{\varepsilon}{ }^{\sigma}$ die Mindestzahl der Terme, die man in (5) berücksichtigen muß, um den Approximationsfehler kleiner als $\varepsilon$ zu machen, so erhält man unter Berücksichtigung der verschiedenen Konvergenzgeschwindigkeiten der Reihen (4) und (5) als Regelfall die Beziehungen

$$
l_{\varepsilon}{ }^{\sigma}>l_{\varepsilon}^{\text {out }}, \quad l_{\varepsilon}^{\text {in }}>l_{\varepsilon}^{\text {out }},
$$

die Ausdruck der wechselseitigen Verschmierung von $I^{\text {in }}(\boldsymbol{n})$ und $\sigma(\cos \Theta)$ sind und im übrigen nicht von der Gültigkeit der zentralen Formel (6) abhängen, da die Entwicklungen (4) und (5) immer möglich sind.

Aus (8) lesen wir für unser Problem ab, daß höchstens $l_{\varepsilon}$ out Differenzen $\sigma_{0}-\sigma_{l}$ aus einem Experiment extrahiert werden können, so daß weder der Grenzwert (7) noch die Reihe (5) bestimmbar sind. Ergänzt man die bestimmbaren $\sigma_{0}-\sigma_{l}$ irgendwie zu einem vollständigen $\sigma(\cos \Theta)$, so muß dessen Unsicherheit nach (8) größer als $\varepsilon$ bleiben. Wir sehen in diesen Aussagen die Präzisierung des Prinzips des Informationsverlustes und der Nichtumkehrbarkeit der Theorie der Kleinwinkelvielfachstreuung und nicht etwa eine Schwäche des benützten Formelapparates, also von (6). Die vorliegende Form der Theorie scheint uns gerade deshalb besonders vorteilhaft, weil sie auf diese Zusammenhänge hinführt und sie nicht verdeckt.

Unsere Antwort auf die eingangs gestellte Frage lautet also: Aus der Messung der Intensitätsverteilung bei Vielfachstreuung läßt sich nur eine mit zunehmendem $N d$ abnehmende Anzahl von Differenzen $\sigma_{0}-\sigma_{l}$ bestimmen, die im Regelfall nicht ausreichen, um daraus den Wirkungsquerschnitt $\sigma(\cos \Theta)$ bestimmen zu können. Eine Bestätigung der Theorie der Kleinwinkelvielfachstreuung durch das Experiment bestätigt das der Theorie zugrunde liegende Einzelstreugesetz nur in den bestimmbaren Differenzen $\sigma_{0}-\sigma_{l}$, so daß mit einer solchen Messung alle $\sigma(\cos \Theta)$ verträglich sind, die in diesen Differenzen übereinstimmen. Zwischen konkurrierenden Ansätzen für $\sigma(\cos \Theta)$, z. B. dem MoLiÈreschen und der zweiten Bornschen Näherung der Coulomb-Streuung ${ }^{5}$, kann daher aus experimentellen Daten bei Vorliegen von Vielfachstreuung nur eine durch die experimentelle Unsicherheit - die experimentellen $\sigma_{0}-\sigma_{l}$ sind mit Fehlern nach dem Fehlerfortpflanzungsgesetz zu versehen! - und durch Informationsverlust eingeschränkte Entscheidung getroffen werden.

Ich danke Herrn Dr. H.-J. Mrister für Diskussionen über den vorliegenden Gegenstand.

5 W. G. Simon, Phys. Rev. 136, B 410 [1964].

\section{Low Energy Electron-Atom Scattering Cross-Section in Thomas-Fermi Theory}

\author{
T. TIETZ \\ Department of Theoretical Physics, University of Lodz, \\ Lodz, Poland \\ (Z. Naturforschg. 21 a, 360-361 [1966] ; received 22 November 1965)
}

In a previous note ${ }^{1}$ the author of this paper has discussed the low energy elastic scattering of electrons by neutral atoms using an approximate potential $V(r)$ for the Hartree field. The purpose of this note is to discuss the low energy electron-atom scattering cross section in Thomas-Fermi theory.

In quantum mechanics the scattering of a particle by a spherically symmetric potential can be described in terms of phase shifts. As known each phase shift is

\footnotetext{
1 T. Tietz, Z. Naturforschg. 19 a, 1413 [1964].

2 R. B. Levy and J. B. Keller, J. Math. Phys. 4, 54 [1963].
}

an odd function of the wave number $k$, where $k$ is related to the energy of the scattered particle by the relation $k^{2}=2 \mathrm{mE} / \hbar^{2}$.

For short range potentials the phase shifts are analytic at $k=0$ and can be expanded in odd powers of $k$. However, the long range potentials are not analytic at $k=0$, so they cannot be expanded in powers of $k$. This difference in low energy expansion of scattering phase shifts for long range potentials has been discussed in great details by Levy and KeLLER ${ }^{2}$. They have shown that the phase shift $\eta_{0}$ and $k \cdot \operatorname{ctg} \eta_{0}$ can be found if the scattering length $a$ is known. In the Levy and Keller method the potential $V(r)$ is a long range potential that behaves asymptotically for large $r$-value as $V(r)=V_{0} r^{-v}$ where $v=4$ and $V_{0}$ is a positive or negative constant. The Thomas-Fermi potential fulfills the required condition of the LEvY-KELLER method. It is a long range potential with the required asymptotic behaviour. We will find below the scattering length $a$ for an approximate Thомаs-Fermi potential. The author of this paper has shown that the following approxima- 